\title{
ESTUDIO ESPACIAL Y TEMPORAL DE LAS TENDENCIAS DE LA LLUVIA EN LA COMUNIDAD VALENCIANA (1961-1990)'
}

\author{
M. DE LUIS (1) \\ J. C. GONZÁLEZ HIDALGO (2) \\ J. RAVENTÓS (1) \\ J. CORTINA (1) \\ J. R. SÁNCHEZ (1)
}

\begin{abstract}
RESUMEN: Hemos analizado las tendencias espaciales $y$ temporales de la magnitud y concentración de las precipitaciones de 96 estaciones pluviométricas de la Comunidad Valenciana ( $E$ España), durante el período normal 1961-1990. Los principales resultados obtenidos son los siguientes:
\end{abstract}

1) Encontramos una tendencia anual de las precipitaciones negativa en el $43 \%$ de las localidades estudiadas; la variabilidad de las cantidades precipitadas se mantiene e incluso se incrementa en el 52\% y $38 \%$ del total de localidades analizadas.

2) La concentración de la lluvia mensual no manifiesta tendencias durante el periodo analizado en el $41 \%$ de las estaciones, mientras se observa un incremento de la misma en el 39\% de localidades. Se observan resultados semejantes en cuanto a su variabilidad.

3) En zonas de precipitación media y baja (sector semiárido) la cantidad de lluvia anual tiende a incrementarse o se mantiene; en las zonas de precipitación media anual más

1. El presente trabajo es el desarrollo in extenso de la primera parte de la comunicación presentada por los autores en la XXII General Assembly of European Geophysical Society celebrada en Viena, durante el mes de abril de 1997.

(1) Departamento de Ecología, Universidad de Alicante (Ap. 99, Alicante, 03080, España).

(2) Departamento de Geografía, Universidad de Zaragoza, (50009, Zaragoza, España). 
elevada (zonas de clima seco y búmedo) predomina la tendencia negativa en el total de precipitación anual.

4) Los resultados los discutimos en el ámbito de las predicciones de los Modelos de Circulación General y los mecanismos generadores de lluvia en la zona de trabajo.

ABSTRACT: We analysed temporal and spatial trends in rainfall volume and concentration from 96 pluviometric stations in the Region of Valencia (E. Spain) from 1961 to 1990. The main results of analysis are the following:

1) We find a decrease in annual rainfall in $43 \%$ of the stations studied. Variability in rainfall volume remains unchanged or increase (52\% and 38\% of the stations respectively).

2) Monthly rainfall concentration shows no temporal trend in $41 \%$ of the stations and a gradual increase in 39\% of the stations. Similar figures are obtained for variability in rainfall concentration.

3) In areas with low average rainfall (semiarid climate), annual rainfall volume increases or remain unchanged, whereas, in areas with bigh average precipitation (dry to subbumid climate) a negative trend dominate.

4) We discuss results in relation to predictions from GCM studies and to the origin of the rainfall events.

Palabras clave: Mediterráneo; tendencias de lluvia; variabilidad de la lluvia; concentración de lluvia; análisis espacial; cambio climático.

Key words: Mediterranean area; rainfall trend; rainfall variability; rainfall concentration; spatial analysis; climatic change.

\section{Introducción}

El cambio climático, como resultado del efecto invernadero por la emisión de gases, es uno de los problemas mundiales más importantes. Las predicciones obtenidas mediante las simulaciones de los Modelos de Circulación General (GCM) sugieren un incremento global y general de la temperatura (Houghton et al., 1995). Sin embargo, las tendencias de la precipitación son menos evidentes. 
Sin lugar a dudas, la disponibilidad de agua es uno de los factores determinantes para controlar la productividad de los ecosistemas terrestres y, por tal razón, los efectos del cambio climático pueden depender en gran medida de los cambios producidos en el ciclo del agua. Esta situación es particularmente evidente en áreas afectadas por procesos de desertificación, como gran parte del sur de Europa (Pérez-Trejo, 1993).

Según las tasas de emisión de gases los GCM predicen un incremento de la precipitación de invierno y un descenso en verano, cifrado entre el $5 \%$ y $15 \%$ (Houghton et al., 1990). El IPCC 1995 indica que los resultados obtenidos de las simulaciones efectuadas para Europa son sensibles al afecto invernadero tanto por emisiones de gas como aerosoles.

Cuando solamente se contempla la incidencia de las emisiones de gas sobre el efecto invernadero, las simulaciones muestran un descenso en la precipitación global de esta área. Si ambos factores se consideran simultáneamente los resultados son contrarios. Finalmente, el nivel de confianza para las escalas regionales es bajo. En esta escala espacial una técnica complementaria de particular interés se basa en el estudio de los registros climáticos. Cuando son analizados mediante series temporales el enfoque llega a ser una poderosa herramienta para detectar tendencias climáticas.

Bradley et al. (1987) y Díez et al. (1989) han estudiado en el Mediterráneo series de precipitación muy prolongadas y han observado un aumento de la lluvia en invierno y primavera junto al descenso de la lluvia de verano y otoño a partir de los años 40. Más recientemente, Loureiro y Coutinho (1995) analizaron dos períodos normales (1931-1990) en 16 estaciones del Algarve (Portugal), y encontraron un incremento significativo de la lluvia anual así como durante el período comprendido entre octubre y febrero.

En este trabajo presentamos los resultados del análisis de las tendencias de 96 estaciones pluviométricas de la Comunidad Valenciana durante el período normal (1961-1990) en la Comunidad Valenciana (E España). Nuestros principales objetivos son la detección de tendencias en el tiempo, en el volumen y concentración de la lluvia, y estudiar su variabilidad. Así mismo estudiamos estas series en relación a su distribución espacial.

Los estudios climáticos en esta zona son particularmente relevantes en la escala local y regional. La Comunidad se encuentra bajo la influencia pluvial tanto del Atlántico como del Mediterráneo (Martín Vide, 1984), y sus lluvias se caracterizan por una elevada concentración asociada a riesgos generalizados de erosión y desertificación (Pérez-Trejo, 1993). 


\section{Metodología}

a) Área de estudio y bases de datos

La Comunidad Valenciana se ubica en la costa mediterránea del Este de España (Fig. 1), entre los $38^{\circ}$ y $40^{\circ}$ de latitud N. El clima es mediterráneo, con elevada estacionalidad de la lluvia a lo que se une una gran variabilidad interanual (véase una descripción general en Peréz-Cueva, 1994).

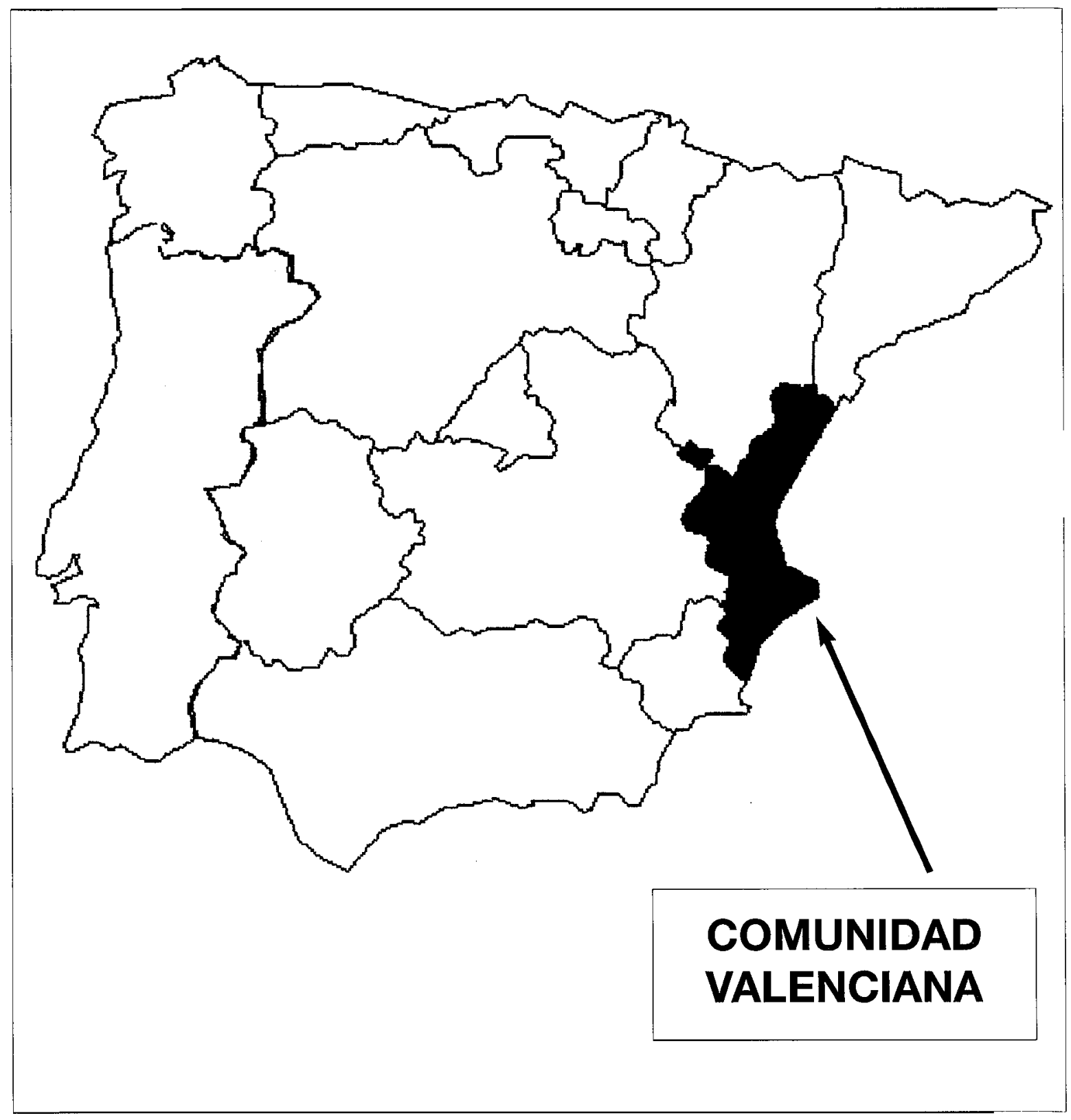

Figura 1.- Localización de la zona de estudio. Comunidad Valenciana. 
En este estudio hemos empleado los datos mensuales de 96 estaciones pluviométricas de la Comunidad Valenciana que abarcan el período normal 19611991 (fuente original: Peréz Cueva, 1994). En cada localidad hemos calculado la media anual de la precipitación (Fig. 2), y la media anual del Índice de Concentración de las Precipitaciones (PCI) descrito por Oliver (1980) (Fig. 3). Cuando los valores del PCI son inferiores a 10 indican una distribución uniforme de la lluvia, mientras que entre 11 y 20 denotan una distribución estacional. Los valores por encima de 20 corresponden a climas con grandes variaciones intermensuales de la precipitación.

\section{b) Análisis temporal}

Antes de someter los datos al análisis de tendencias, los registros fueron suavizados al objeto de reducir la variabilidad natural; este análisis se efectuó mediante el cálculo de la media móvil de intervalo de 9 años. Según Sneyers (1992) el efecto de este suavizado artificial sugiere la evolución climática que es independiente de componentes no aleatorios. Finalmente, el estudio de la significación del valor de la tendencia (positiva, negativa o nula) se ha realizado mediante la prueba no paramétrica de rangos de Spearman (con nivel de significación 5\%) nuevamente aconsejada por Sneyers (1992). Las variables incluidas en este análisis fueron: el tiempo, el rango de la precipitación anual (cantidad) y su variabilidad (PCI). Siegel (1956) ha demostrado que la potencia de la prueba de Spearman representa el $90 \%$ de la potencia que resulta del análisis con el coeficiente de correlación de Pearson (r) en un mismo nivel de significación. El citado análisis se desarrolló en cada una de las 96 estaciones.

Junto al análisis citado hemos estudiado la tendencia de los residuos al objeto de observar los cambios en la variabilidad entre años (predictibilidad), tanto de la cantidad de lluvia anual como de la concentración. El método seguido ha sido el ya comentado, pero los residuos fueron contemplados como coeficientes de variación (CV) para evitar incrementos potenciales de la variabilidad con el incremento de los valores promedio. De esta manera, las variables incluidas en este segundo análisis fueron, además del tiempo, el rango de las siguientes variables: volumen de lluvia anual (ARV), coeficiente de variación anual de la lluvia (CVARV), PCI anual (APCI) y coeficiente de variación anual del PCI (CVAPCI).

\section{c) Análisis espacial}

Hemos aplicado la prueba no paramétrica de Cramér-von Mises (Syrjala, 
1996) para comparar la distribución espacial del signo de la tendencia de las cuatro variables citadas de las 96 estaciones.

Para las cuatro variables -signo de la tendencia de ARV (Fig. 4), CVARV (Fig. 5), APCI (Fig. 6) y CVAPCI (Fig. 7)- definimos tres diferentes poblaciones (localidades con tendencia positiva, negativa y nula) y se construyó una función de distribución para cada una de las tres poblaciones agrupadas por dos criterios diferentes de clasificación: la media de precipitaciones anuales (Fig. 2) y la media del valor de PCI (Fig. 3). En relación a ambos criterios hemos comprobado si las dos poblaciones se solapaban (Tabla 2a).

El análisis se desarrolló en todos los pares posibles de poblaciones.

La hipótesis nula que se consideró era que a lo largo del territorio de la Comunidad Valenciana la distribución normalizada de cada par de poblaciones de signos diferentes era la misma; como hipótesis alternativa se consideró que había diferencias no específicas en las distribuciones citadas. En nuestro caso las poblaciones son representadas por el signo de la tendencia.

Cada pareja de funciones de distribución empíricas acumuladas fue comparada con el estadístico $Y$ construido para ambos criterios de clasificación ( $\Psi$ prep y $\Psi$ pci). El nivel de significación del estadístico fue determinado mediante el empleo de una prueba de aleatoriedad (Edgington, 1980). Este modelo univariado no depende del punto de comienzo de cálculo de la función de distribución acumulada.

\section{Resultados}

\section{a) Distribución espacial y temporal de las tendencias de la lluvia}

Del conjunto de las 96 localidades estudiadas 11 presentan una tendencia de la lluvia anual positiva; 43 localidades tienen tendencia negativa y 42 no tienen tendencia detectada $(\mathrm{p}<0.05)$ (Tabla 1). La distribución en el territorio de estas tendencias se muestra en la Figura 4. En general en la zona sur los valores de las tendencias son positivos o nulos, mientras que en el centro y norte de la Comunidad son negativos o nulos. Existe una agregación de las tendencias negativas en la zona de costa. Esta pauta espacial mostrada en la Figura 4 se solapa con la distribución en el espacio de la precipitación media anual (Fig. 2). 


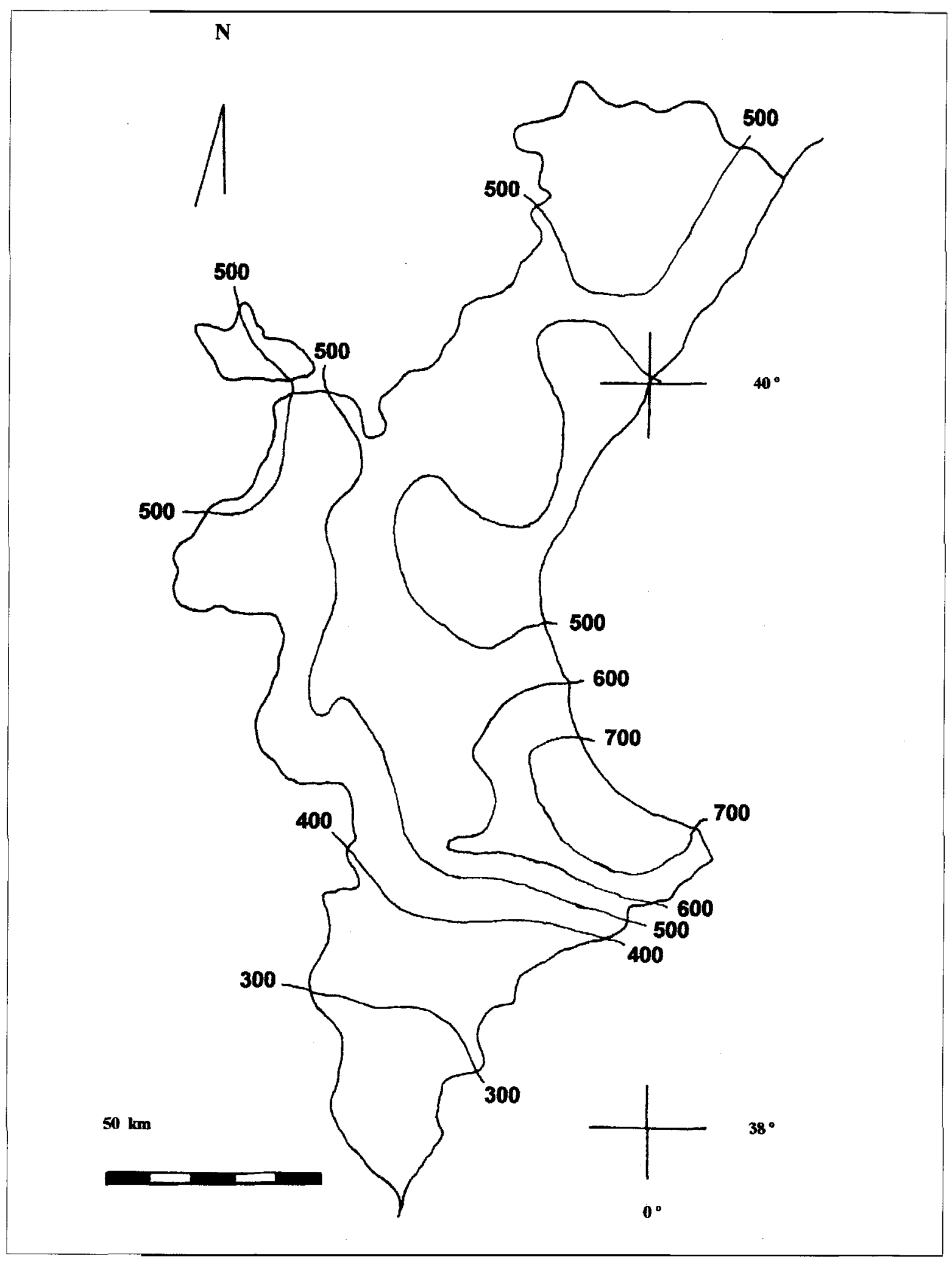

Figura 2.- Precipitación media anual (mm) en la Comunidad Valenciana (1961-1990), en Pérez Cueva (1994) simplificado. 
M. DE LUIS - J. C. GONZÁLEZ HIDALGO - J. RAVENTÓS - J. CORTINA - J. R. SÁNCHEZ

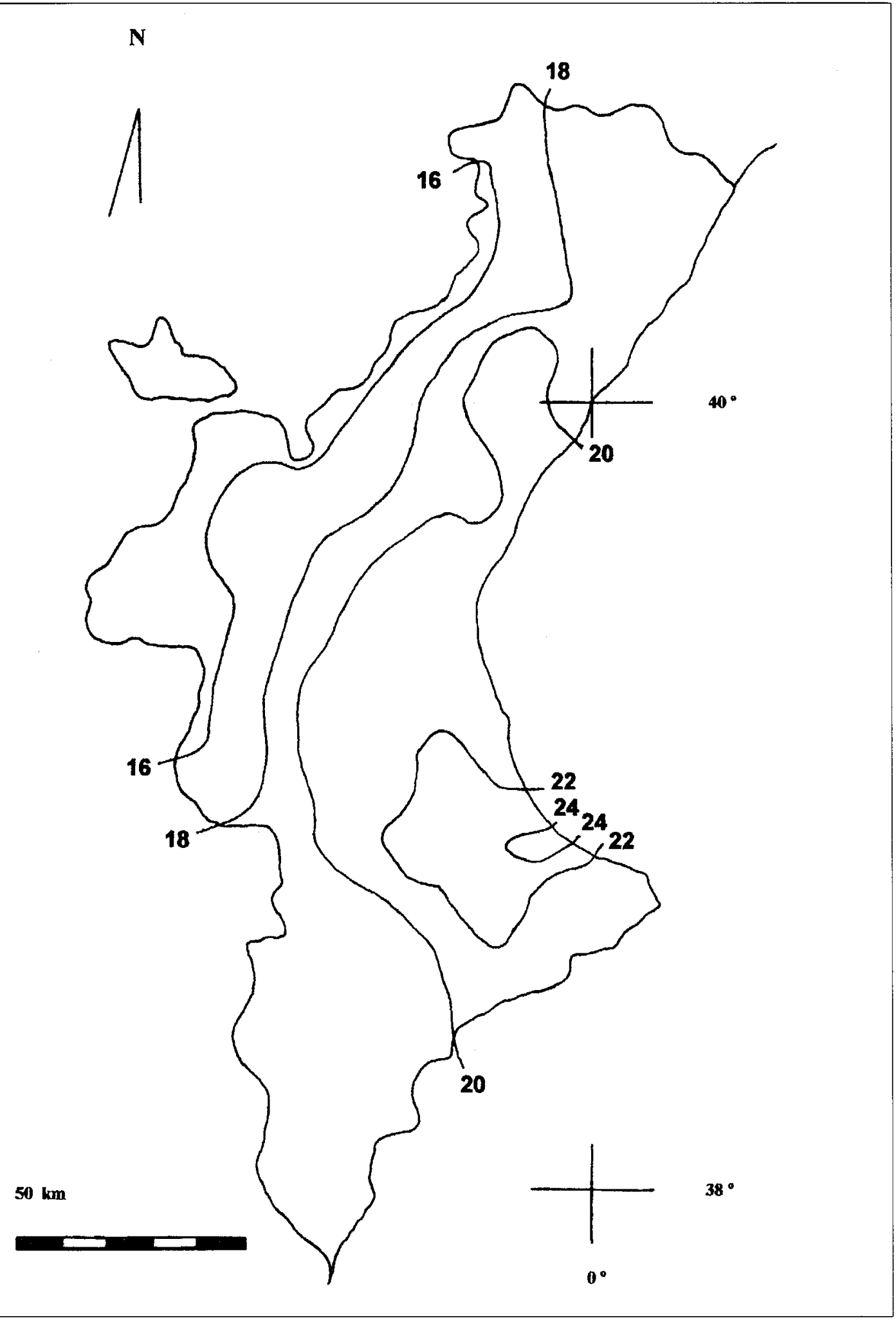

Figura 3.-Índice de Concentración de las Precipitaciones (Oliver, 1980) en la Comunidad Valenciana (1961-1990). 
Cuando se comparan la distribución espacial de las tendencias del volumen (ARV) con el estadístico de clasificación Uprep, cada pareja de poblaciones de signos no se solapan (Tabla 2a). Ello quiere decir que en áreas de alta precipitación media anual (clima seco y subhúmedo) la tendencia predominante es negativa; en las zonas con precipitación media anual intermedia la tendencia es predominantemente negativa o acaso nula, mientras que, finalmente, en zonas de baja precipitación media anual (clima semiárido mediterráneo) la tendencia predominante es positiva o nula.

Los resultados del análisis de la tendencia de la variabilidad (CVARV) se muestran en la Tabla 1. La distribución de la misma es algo distinta (Fig. 5) a la obtenida en el volumen de precipitación (Fig. 4). Todas las comparaciones pareadas de las poblaciones diferenciadas por signos de tendencia están significativamente desagrupadas según el estadístico $\Psi$ prep (Tabla 2a). Como se muestra en la Fig. 5, no se detectan tendencias dominantes en las localidades del sur (precipitación media anual baja), mientras que en el centro y norte del territorio de la Comunidad la tendencia generalizada es positiva o nula. La variabilidad, y por tanto la impredictibilidad hacia el futuro tiende a incrementarse en aquellas estaciones de elevada precipitación localizadas en las cercanías de la costa.

Tabla 1.- Número de estaciones pluviométricas según la tendencia de las variables estudiadas.

\begin{tabular}{|c|c|c|c|}
\hline \multirow{2}{*}{ Variables pluviométricas } & \multicolumn{3}{|c|}{ Número de estaciones } \\
\cline { 2 - 4 } & $\begin{array}{c}\text { Positivo } \\
(+)\end{array}$ & $\begin{array}{c}\text { Negativo } \\
(-)\end{array}$ & $\begin{array}{c}\text { Nulo } \\
(\mathrm{o})\end{array}$ \\
\hline Precipitación media anual (ARV) & 11 & 43 & 42 \\
\hline CV de la lluvia anual (CVARV) & 38 & 6 & 52 \\
\hline PCI anual (APCI) & 39 & 16 & 41 \\
\hline CV del PCI anual (CVAPCI) & 25 & 16 & 55 \\
\hline
\end{tabular}

b) Distribución temporal y espacial de la concentración de la lluvia.

La distribución espacial de la concentración de la lluvia manifiesta un gradiente desde la costa hacia el interior (Fig. 6), cuyo máximo corresponde aproximadamente con el máximo del volumen anual de lluvia (Fig. 3). Los valores del índice PCI están cercanos a 20 en gran parte del territorio de la Comunidad, y alcanzan un máximo de 25 . El número de estaciones con diferente signo de tendencia se muestra en la Tabla 1.

Al comparar la distribución en el espacio de la tendencia anual del índice PCI con el criterio $\Psi$ pci, los diferentes grupos de estaciones agrupadas por signos no se solapan (Tabla 2b). Las localidades con valores de PCI $<20$ (sobre todo en el interior) muestran un incremento general de la concentración de la 
lluvia durante el período 1961-1990, mientras que las zonas con altos valores de concentración de lluvia (PCI $>20)$ predominantemente tienen tendencias negativas o nulas.

Las tendencias de la variabilidad de la concentración de la lluvia (CVAPCI) han resultado en 25 estaciones positivas y en 16 localidades las tendencia son negativas de manera que la mayoría de las mismas tiene tendencia nula (55) (Tabla 1). La distribución espacial de esta variable (Fig. 7) no ha resultado significativa respecto al estadístico $\Psi$ pci. Como consecuencia, no se detectan áreas diferenciadas según la tendencia de la variabilidad de la concentración de la lluvia anual.

Tabla 2.- Estudio de la distribución espacial de las tendencias de las variables estudiadas. Los valores significativos $(P<0.05)$ se indican en cada pareja de poblaciones e indican que ambas no se solapan entre sí en un particular gradiente espacial ( $\Psi$ prep, $\Psi$ pci). Las tendencias pueden ser positivas $(+)$, negativas $(-)$ o nulas $(\Leftrightarrow)$. Explicaciones más detalladas se ofrecen en el texto. (a) Comparación de pares de tendencias de la lluvia anual (cantidad de lluvia

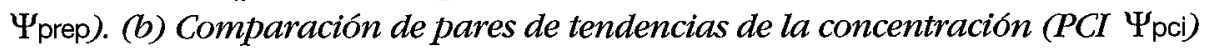

TABLA 2a

\begin{tabular}{|c|c|c|}
\hline \multirow[t]{2}{*}{ Variables pluviométricas } & \multicolumn{2}{|c|}{$\begin{array}{l}\text { Grado de significación } \\
\text { en las comparaciones } \\
\text { a pares para cada } \Psi \text { prep } \\
\text { criterio de clasificación }\end{array}$} \\
\hline & & $\bar{P}$ \\
\hline Volumen de lluvia anual (ARV) & $\begin{array}{l}\text { + vs - } \\
\text { + vs o } \\
\text { - vs o }\end{array}$ & $\begin{array}{l}0.003 \\
0.049 \\
0.002\end{array}$ \\
\hline CV de la lluvia anual (CVARV) & $\begin{array}{l}+\mathrm{VS}- \\
+\mathrm{cs} O \\
-\mathrm{VSO}\end{array}$ & $\begin{array}{l}0.001 \\
0.002 \\
0.029\end{array}$ \\
\hline
\end{tabular}

TABLA 2b

\begin{tabular}{|c|c|c|}
\hline \multirow{2}{*}{ Variables pluviométricas } & \multicolumn{2}{|c|}{$\begin{array}{c}\text { Grado de significación } \\
\text { en las comparaciones } \\
\text { a pares para cada } \Psi \text { prep } \\
\text { criterio de clasificación }\end{array}$} \\
\hline \multirow{2}{*}{ PCI anual (APCI) } & & P \\
\cline { 2 - 3 } & + vs - & 0.001 \\
& + vs o & 0.001 \\
& - vs o & 0.001 \\
\hline \multirow{2}{*}{ CV del PCI anual (CVAPCD) } & + vs - & ns \\
& + cs o & ns \\
& - vs o & ns \\
\hline
\end{tabular}




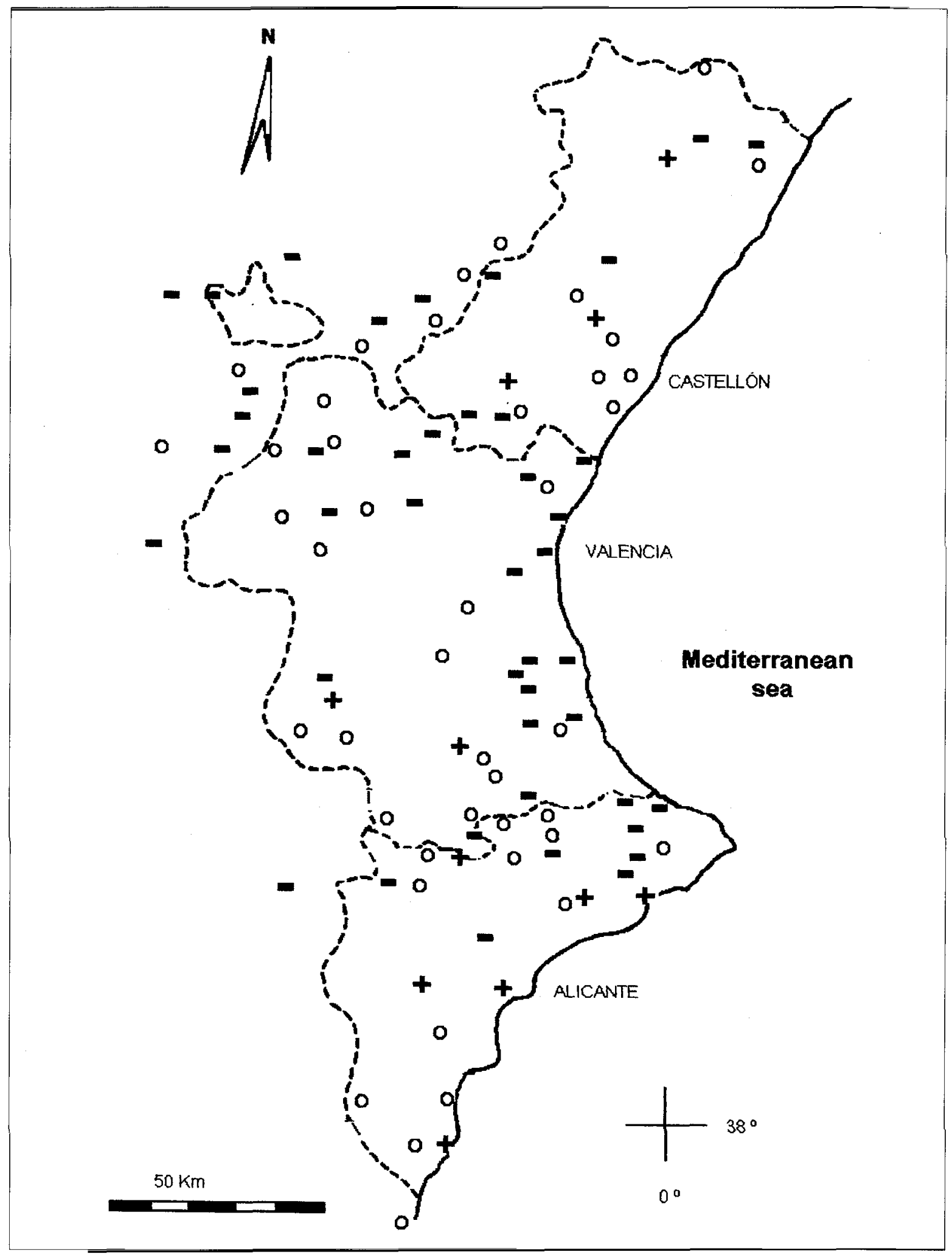

Figura 4.- Tendencias de la precipitación anual (ARV) en la Comunidad Valenciana (1961-1990). Las tendencias pueden ser positivas $(+)$, negativas $(-)$ o nulas $(o)$. 


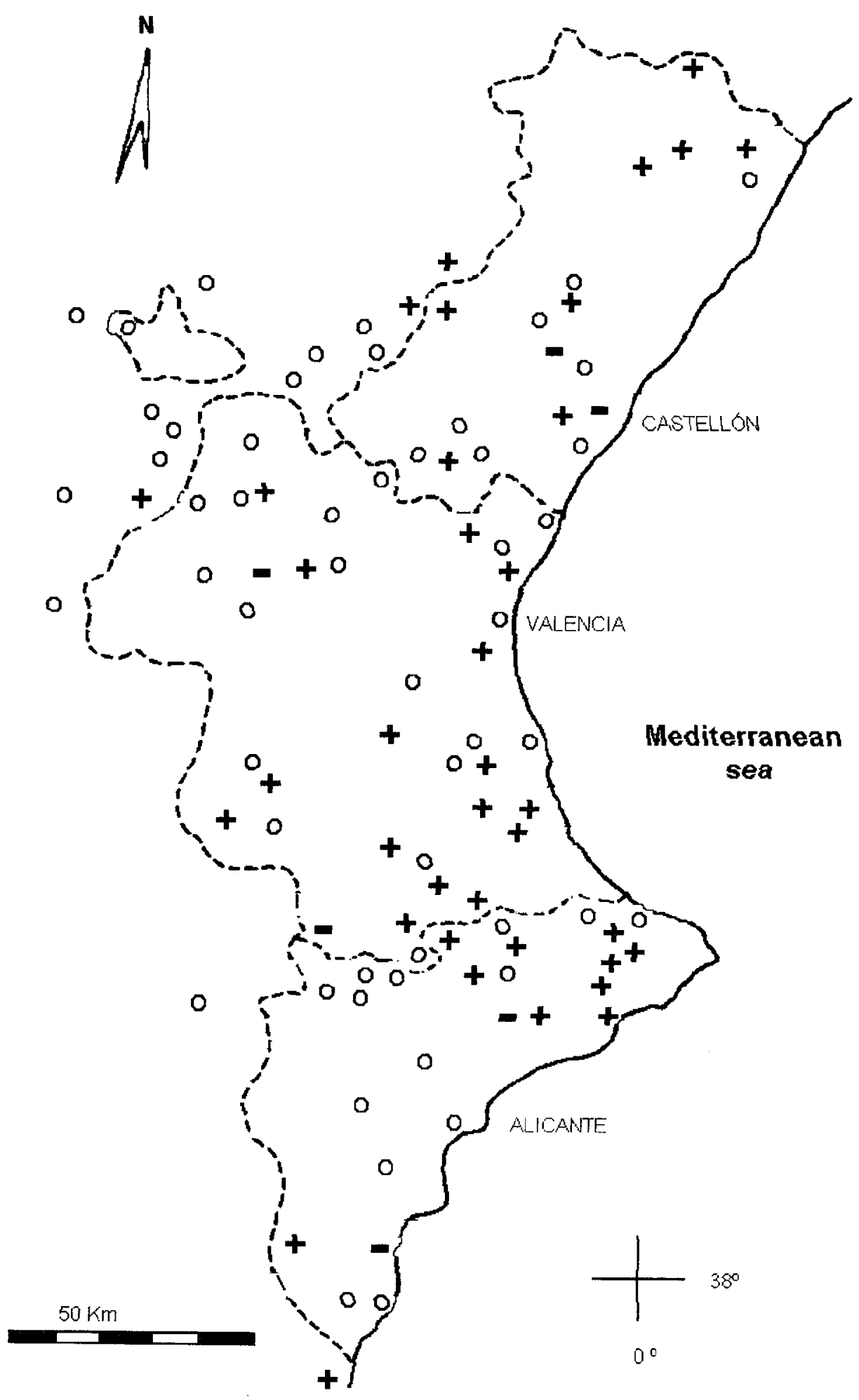

Figura 5.- Tendencias en el coeficiente de variación de la precipitación anual (CVARV) en la Comunidad Valenciana (1961-1990). Las tendencias pueden ser positivas $(+)$, negativas $(-)$ o nulas (o). 


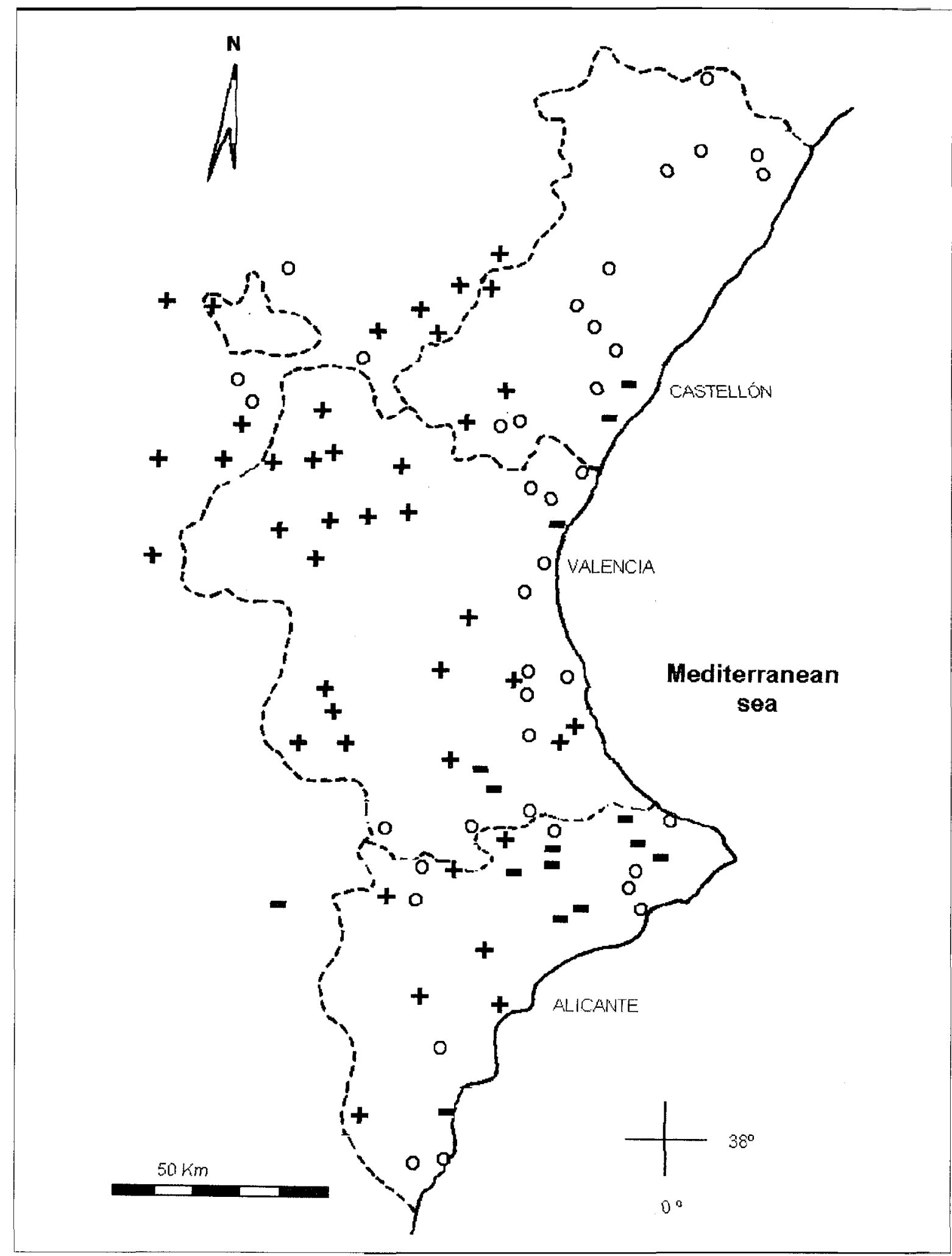

Figura 6.- Tendencias en el Índice de Concentración de las Precipitaciones (APCI) en la Comunidad Valenciana (1961-1990). Las tendencias pueden ser positivas $(+)$, negativas $(-)$ o nulas (o). 


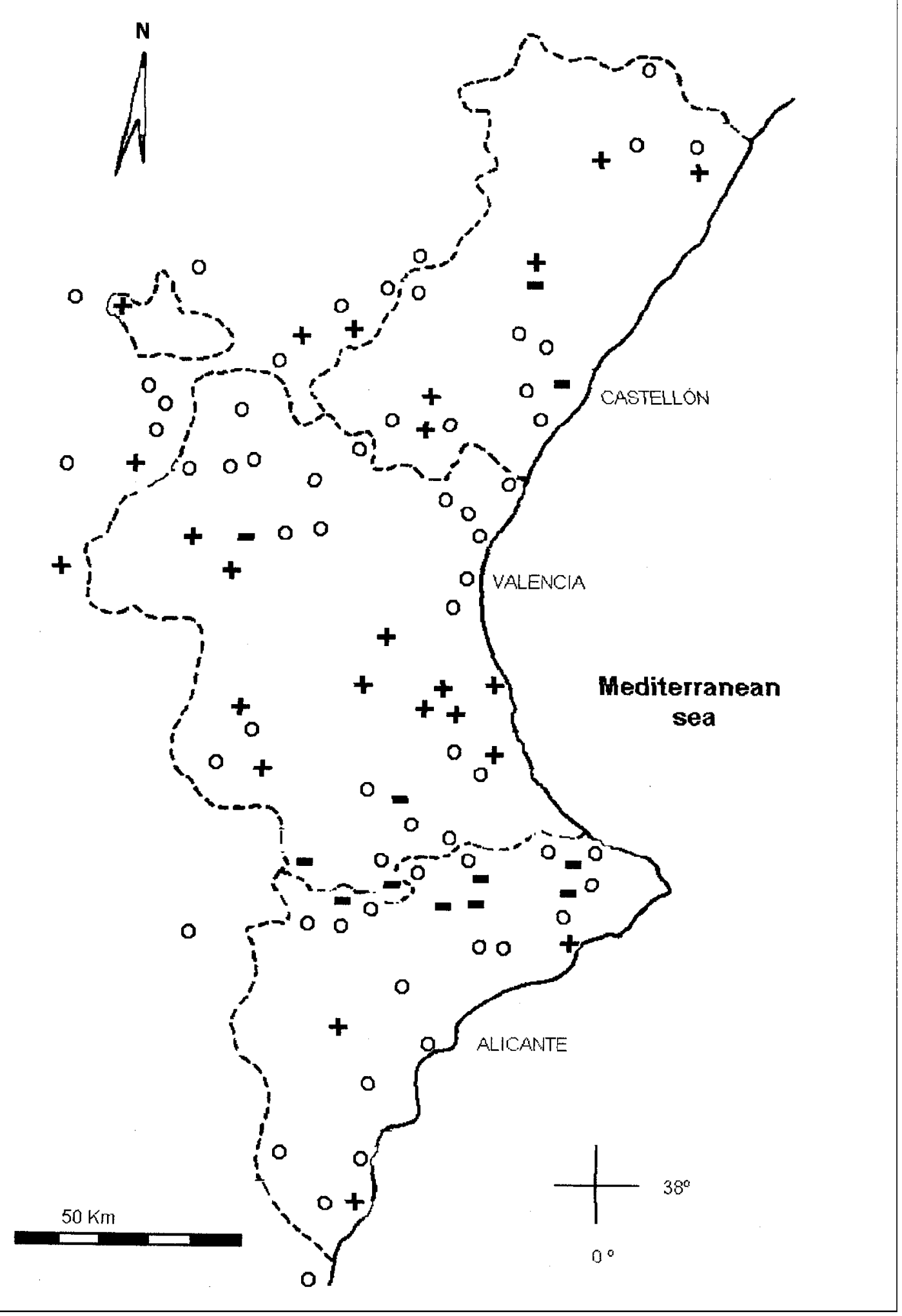

Figura 7.- Tendencias en el coeficiente de variación del Índice de Concentración de las Precipitaciones (CVAPCD en la Comunidad Valenciana (1961-1990). Las tendencias pueden ser positivas $(+)$, negativas $(-)$ o nulas $(0)$. 


\section{Discusión}

La precipitación en la Comunidad Valenciana parece haber cambiado a lo largo del período de 30 años comprendido entre 1961-1990. Junto a un descenso en la cantidad anual de lluvia se ha producido un aumento de su variabilidad en la mayoría de las estaciones analizadas. Estos resultados concuerdan con las predicciones de GCM relativas al descenso futuro de la precipitación y el incremento de su variabilidad en el ámbito mediterráneo (Palutikof, 1996a). Los cambios estacionales (incremento de la lluvia invernal y descenso en verano) han sido predichos en Europa del sur (IPCC 1990, 1995), y hay también indicaciones para las áreas mediterráneas del descenso general de la precipitación desde los años treinta (Maheras, 1988; Amanatidis, 1993). El acuerdo entre tales predicciones y las observaciones realizadas sugiere que esta tendencia puede estar ligada al calentamiento global (Palutikof et al., 1996b).

El aumento en la variabilidad de la precipitación observado en la Comunidad Valenciana concuerda con los estudios que han analizado las relaciones entre los campos de presión del Atlántico Norte, al nivel del mar, y las precipitaciones invernales de la Península Ibérica a lo largo de la última centuria (von Storch et al., 1993). En estas investigaciones se ha encontrado un descenso de la precipitación media de invierno en torno a los $7 \mathrm{~mm}^{\mathrm{mes}^{-1}}$ con una amplitud de la variabilidad interanual de $\pm 10 \mathrm{~mm} \mathrm{mes}^{-1}$.

La tendencia general observada se corresponde a la indicada en las áreas de influencia atlántica. Malberg y Bökens (1993) han observado en la Península Ibérica aumentos de la lluvia de invierno y descensos en verano; Loureiro y Coutinho (1995) han detectado un aumento de la precipitación anual en el sur de Portugal. En general, todos estos cambios pueden estar relacionados con las tendencias particulares de las diferentes estaciones climáticas, así como con las tendencias de los gradientes de presión (Malberg y Bökens, 1993; Flohn, 1993a, 1993b).

Según el origen de las precipitaciones, el territorio de la Comunidad de Valencia ha sido dividido en dos áreas contrastadas (Martín Vide, 1984). Las áreas semiáridas del sur, bajo la influencia atlántica, donde las borrascas procedentes del Atlántico vía estrecho de Gibraltar son la principal fuente de precipitación (corresponde prácticamente a la totalidad de la provincia de Alicante), y el sector central y norte donde la precipitación resulta principalmente de fenómenos convectivos originados sobre el Mediterráneo. Según este patrón global, la cantidad de las precipitaciones de las áreas semiáridas ha permanecido estable o incluso se ha incrementado en el período de estudio (1961-1990). En nuestro análisis no hemos detectado cambios en la concentración o variabilidad en toda esta zona. Quizá los incrementos que se han sugerido para estas 
áreas en la lluvia de invierno y el descenso de la de verano puedan compensarse entre sí para no modificar la tendencia anual.

Por su parte, las zonas dominadas por las precipitaciones de origen mediterráneo pueden subdividirse en dos conjuntos, que reflejan el gradiente señalado por Camarasa (1993). El primero corresponde a la zona costera, sector en el que se ha detectado una tendencia del volumen de lluvia anual negativa a la vez que presenta una tendencia positiva de su variabilidad. En este sector no hemos observado en el período de estudio tendencias significativas de la concentración de la lluvia. El segundo subsector (centro interior y norte) muestra pautas semejantes en la tendencia anual de la precipitación y su variabilidad interanual, pero las tendencias no han sido tan evidentes como en el caso previo; por el contrario, la concentración de la lluvia ha aumentado en muchas estaciones principalmente en las localidades del interior.

Los patrones de distribución de la concentración observados en este estudio son semejantes a los obtenidos en análisis previos en toda la costa mediterránea española (Martín Vide, 1984) o para la propia Comunidad Valenciana (De Luis, 1996).

La magnitud de la concentración de la lluvia en el conjunto de la Comunidad, y especialmente en las zonas costeras es elevada e indica diferencias substanciales en los totales mensuales. Así mismo podemos señalar que en muchas zonas de la Comunidad la recurrencia del fuego, un segundo factor clave en el riesgo de desertificación, es muy alta (Abad et al., 1996). Si consideramos que la concentración de la lluvia es un factor relevante que afecta su capacidad erosiva (González Hidalgo, 1996), nuestros resultados ponen de manifiesto el elevado riesgo de desertificación que padece esta área (PérezTrejo, 1993; López Bermúdez, 1990).

En resumen, el análisis de las tendencias temporales del volumen de precipitación y concentración, junto al estudio de su variabilidad, revela diferencias espaciales significativas no existiendo un patrón global para toda la Comunidad Valenciana. Tales diferencias parecen estar relacionadas con el origen de los mecanismos generadores de las precipitaciones y el gradiente costa interior observado.

\section{Agradecimientos}

Los autores manifiestan su agradecimiento al Dr. Pérez Cuevas (Dep. Geografía, U. Valencia) así como al Gobierno Regional de la Comunidad Valencia (Conselleria d'Ordenació del Territori, Generalitat Valenciana) por las facilidades dadas para acceder a las bases de datos empleadas en el presente estudio. Este trabajo ha sido financiado por CICYT, Proyecto CLI95-1947-CO3-O3. 
ESTUDIO ESPACIAL Y TEMPORAL DE LAS TENDENCIAS DE LA LIUVIA EN LA COMUNIDAD VALENCIANA (1961-1990)

\section{Referencias bibliográficas}

Abad, N.; Caturla, R.; Baeza, J.; Bladé, C.; Vieira, F.; Carbó, E.; Valdecantos, A.; Bonet, A.; Serrasolsas, I.; Guàrdia, R.; Raventós, J.; Alloza, J.A.; Escarre, A.; BELLOT, J. Y VALLEJO, V.R. (1996) Regeneración de los montes quemados. En: V.R. VAllejo (Ed.) (1996) La Restauración de la Cubierta Vegetal en la Comunidad Valenciana: 52148. CEAM-Generalitat Valenciana. Valencia.

Amanatidis, G.T; Paliatsos A.G.; Repapis, C.C. y BarTzis, J.C. (1993) Decreasing precipitation trend in the Marathon area, Greece.

Bradley, R.S.; Díaz, H.S.; Eischeid, J.K.; Jones, P.D.; Kelly, P.M. y Goodess, C.M. (1987) Precipitation fluctuations over northern hemisphere land areas since the mid-19th Century. Science, 237: $171-175$.

Camarasa, A. (1993) La estructura interna de las tormentas mediterráneas. Cuadernos de Geografía, 54: 169-188.

De Luis, M.; GonZÁlez-Hidalgo, J.C. y SánCheZ, J.R. (1996) Análisis de la distribución espacial de la concentración diaria de precipitaciones en el territorio de la Comunidad Valenciana. Geografía Física, 59: 47-62.

DÍAZ, H.S.; BRADleY, R.S. y EISCHEID, J.K. (1989) Precipitation fluctuations over global land areas since the late 1800's. J. Geophys. Res; 94 (1): 1195-1210.

Edgington, E.S. (1980) Randomization test. Second edition. Marcel DeKKer, New York, USA.

FloHn, H. (1993 a) Klimaprobleme vor und nach der Rio-Konferenz (Juni 1992), preprint.

FloHn, H.; (1993 b) Physical 3D-Climatology from Hann to the Satellite Era. Interactions Between Global Climate Subsystems, The Legacy of Hann. Geophys. Monograph 75, IUGG Volume 15.

FlohN, H.; KAPALA, A.; KNOCHE, H.R. Y MÄCHEL, H. (1992) Water vapours as amplifier of the greenhouse effect: New aspects. Meteorol. Z.N.F,1: 122138.

GonZÁlez Hidalgo, J.C. (1996) Los índices de agresividad de la lluvia y su aplicación a la valoración de la erosión del suelo. Cuadernos Técnicos 10, Soc. Española de Geomorfología, Logroño.

Houghton, J.T.; Jenkins, G.J. y Ephraums, J.J. (Eds.) (1990) Climate Change. The IPCC Scientifc Assessment. Policymakers Summary. Cambridge Univ. Press. Cambridge.

Houghton, J.T.; Meira Filho, L.G.; Callander, B.A.; Harris, N.; Kattenberg, A. Y MASKELL, K. (Eds.) (1995) Climate Change 1995. The Science of Climate Change. Policymakers Summary. Cambridge Univ. Press. Cambridge.

LÓPEZ BERMúdez, F. (1990) La degradación de tierras en ambientes áridos y 
semiáridos. Causas y consecuencias. En T. LAsanta Y J.M. García-RuIz (Eds.) Erosión y Recuperación de Tierras Marginales: 51-72. Instituto de Estudios Riojanos. Logroño.

LOUREIRO, N.S. y CoUTINHO, M.A. (1995) Rainfall changes and rainfall erosivity increase in the Algarve (Portugal). Catena 24: 55-67.

MaHeras, P. (1988) Changes in precipitation conditions in the western Mediterranean over the last century. J. Climatol., 8: 179-189.

MAlberg, H. Y BöKEns, G. (1993) Anderungen im Druck-/Geopotential- und Temperatur-gefälle zwischen Subtropen und Subpolarregion im atlantischen Bereich im Zeitraum 1960-90. Meteorol. Z.N.F., 2: 131-137.

MARTín VIDE, J. (1984) Análisis de la irregularidad de la precipitación diaria en el litoral mediterráneo de la Península Ibérica. Rev. Geofísica, 40: 101-106.

OLIVER, J.E. (1980) Monthly precipitacion distribution: a comparative index. Professional Geogr., 32, 3: 300-309.

PALUTIKof, J.P. (1996 a) Scenarios of future rainfall over the Mediterranean: Is the region drying? Book of Abstracts. International Conference on Mediterranean Desertification.

PALUTIKof, J.P; GoOdess, C.M. Y HACOT, H. (1996 b) Future rainfall trends over the Mediterranean. Book of Abstracts. International Conference on Mediterranean Desertification.

Pérez Cueva, A. (Dir) (1994) Atlas climàtic de la Comunitat Valenciana. Direc. General d'Urbanisme i Ordenació del Territori, Generalitat Valenciana, Col.leció Territori, N. ${ }^{\circ} 4$.

PÉRez-Trejo, F. (1994) Desertification and Land Degradation in the European Mediterranean. EC EPOCH Programme. Report EUR 14850. Luxembourg.

SIEGEL, S. (1956) Nonparametric Statistics for the behavioural Sciences. McGraw-Hill, New York.

SNEYERS, R. (1992) Use and Misure of Statistical Metbods for the Detection of Climate Change. Report on the Informal Planning Meeting of Statistical Procedures for Climate Change Detection. World Climate Data Programme Series 20, Annex 3, WMO.

SyRJALA, S.E. (1996) A statistical test for a difference between the spatial distribution of two populations. Ecology, 77, 1: 75-80.

vON STORCH, H.; ZORITA, E. Y CUBASCH, U. (1993) Downscaling of Global Change estimates to regional scales: An application to Iberian rainfall in wintertime. J. of Climate, 6: 1161-1171.

Zimmerman, D.L. (1993) A bivariate Cramér-von Mises type of test for spatial randomness. Applied Statistics 42: 43-54. 